Service social

\title{
Questions et perspectives de recherche concernant la pauvreté chez les jeunes
}

\section{Madeleine Gauthier, Andrée Roberge et Louise Saint-Laurent}

Volume 44, numéro 3, 1995

Pauvreté

URI : https://id.erudit.org/iderudit/706706ar

DOI : https://doi.org/10.7202/706706ar

Aller au sommaire du numéro

Éditeur(s)

École de service social de l'Université Laval

ISSN

1708-1734 (numérique)

Découvrir la revue

Citer cet article

Gauthier, M., Roberge, A. \& Saint-Laurent, L. (1995). Questions et perspectives de recherche concernant la pauvreté chez les jeunes. Service social, 44(3), 55-70. https://doi.org/10.7202/706706ar
Résumé de l'article

Le nombre de jeunes pauvres, âgés de 16 à 24 ans, qui vivent seuls de façon provisoire ou durable, est en progression rapide au Québec. Cette période de la vie est traversée aujourd'hui par de multiples paradoxes allant d'un désir d'autonomie précoce, fondé sur des valeurs modernes d'affirmation de soi et de liberté, à l'allongement de la dépendance, surtout économique, dans un contexte social en mutation. Si les statistiques renseignent sur les caractéristiques de la pauvreté, telles qu'établies à partir des seuils de faible revenu, elles ne permettent toutefois pas de saisir la complexité des situations, des représentations et des stratégies correspondantes. Le rappel de ces données sera suivi de quelques pistes de réflexion qui pourront alimenter éventuellement la recherche et l'action. 


\title{
Questions et perspectives de recherche concernant la pauvreté chez les jeunes
}

\author{
Madeleine GAUTHIER \\ Professeure, INRS-Culture et société \\ Andrée ROBERGE \\ Professeure, Université Laval \\ Louise SAINT-LAURENT \\ Associée de recherche, INRS-Culture et société
}

II va de soi qu'un numéro thématique consacré à la pauvreté ne peut ignorer la situation des jeunes à cet égard. Beaucoup de choses ont déjà été dites ${ }^{1}$; beaucoup de sous-entendus subsistent. La première partie de l'exposé rappellera quelques données administratives faisant état des caractéristiques de la pauvreté telles qu'établies à partir des seuils de faible revenu qui tiennent lieu de définition de la pauvreté au Canada. Trois dimensions seront retenues: les taux de pauvreté, $c^{\prime}$ est-à-dire la proportion de personnes pauvres dans le groupe de référence; l'écart par rapport au seuil, ce qui donne une certaine idée de la gravité de la situation; enfin, le type de ménage auquel appartient la personne qui doit se contenter d'un faible revenu pour assurer sa subsistance. 
II sera plus particulièrement question des $16-24$ ans, cet âge correspondant à la fin de l'obligation scolaire et à la période d'insertion sociale et professionnelle. Cette période de la vie est traversée aujourd'hui par de multiples paradoxes allant d'une autonomie précoce en ce qui touche certaines dimensions de la vie, dont la vie affective, à l'allongement de la dépendance ailleurs, en particulier de la dépendance économique (Gauthier, 1994).

La deuxième partie soulèvera un certain nombre de questions quant aux connaissances que nous avons de la réalité qui se trouve derrière les données officielles. Ces questions pourraient dessiner de la sorte quelques avenues pour la recherche. Si, historiquement, les états démocratiques $n^{\prime}$ ont jamais été aussi à même de prendre le pouls de leur population, leurs ressortissants ne se reconnaissent pourtant pas toujours dans ces informations rendues disponibles par les administrations publiques. Simples citoyens comme praticiens des sciences sociales confrontent avec les statistiques d'État leur perception d'une réalité complexe et multiple, une vision du "réel » construite à partir des observations, des expériences du quotidien et des échanges avec l'entourage. Ces visions issues du "sens commun » et des limites de l'expérience individuelle ne sauraient bien sûr rivaliser avec ces clichés photographiques que constituent les statistiques d'État. Elles leur donnent du relief et souvent les remettent en question. Cette partie de l'exposé consistera à suggérer quelques autres pistes dans la manière d'aborder la question de la pauvreté chez les jeunes, en particulier les parcours qui mènent à la précarité financière et les stratégies utilisées pour préserver son intégrité physique, psychologique et matérielle ${ }^{2}$. Enfin, quelques voies théoriques qu'emprunte la recherche actuelle pourront suggérer des manières différentes d'interroger la réalité, de la catégoriser et d'indiquer les chemins de l'intervention.

\section{LES DONNÉES ACTUELLES}

En 1993, le Conseil permanent de la jeunesse attirait l'attention sur le groupe des 15-30 ans. "Dites à tout le monde qu'on existe", titrait un avis fondé sur des statistiques et une enquête sur la pauvreté des jeunes. II s'agit d'un groupe d'âge fortement atteint si l'on tient compte des données recueillies selon la manière habituelle de mesurer la pauvreté au Canada, soit l'établissement d'un seuil de faible revenu à partir d'une enquête effectuée par Statistique Canada sur les finances des consommateurs. Ce seuil tient compte de la composition du ménage et de la taille de la localité d'habitation. 
L'accentuation du phénomène de la pauvreté chez les jeunes date du début des années 80 , période où les taux de chômage chez les jeunes ont été particulièrement élevés. Jusque-là, la pauvreté renvoyait davantage aux personnes âgées, aux femmes seules avec des enfants ou à des veuves dans la cinquantaine. Diverses mesures - pensions de vieillesse, régimes de retraite ou autres assurances - ont fait en sorte que ces groupes présentent aujourd'hui un taux de pauvreté moins élevé ou encore un indice de gravité moins grand que ce qu'on trouve chez les plus jeunes et, en particulier, chez les jeunes qui vivent seuls. II existe encore beaucoup de pauvres chez les personnes de 65 ans et plus, mais l'écart entre la moyenne des revenus à cet âge et le seuil de faible revenu est moins accentué (Conseil national du bien-être, 1995 : 46).

\section{Les taux de pauvreté sont particulièrement élevés chez les jeunes}

En 1990, selon une compilation spéciale du ministère de la Sécurité du revenu et de la Formation professionnelle (actuellement le ministère de la Sécurité du revenu) établie à la demande du Conseil permanent de la jeunesse, $39,2 \%$ des moins de trente ans qui vivent seuls et $17,1 \%$ de l'ensemble des jeunes familles se trouvaient sous le seuil de faible revenu. Les jeunes familles pauvres se composent à $83,9 \%$ de familles monoparentales, $12,9 \%$ de familles biparentales et $7,3 \%$ de couples sans enfants (1993:11).

La composition des ménages pauvres chez les jeunes est sans doute très variée. Il faudrait une étude minutieuse de ce groupe particulier pour en connaître tous les visages. Par exemple, les étudiants obligés de quitter leur famille pour poursuivre leurs études sont sans doute classés parmi les pauvres, puisque peu parmi eux ont le revenu moyen des personnes seules aptes au travail qui était, au Québec, de 22527 \$ en 1994 (Conseil national du bien-être social, 1995 : 32). Cette situation temporaire de pauvreté est généralement acceptée, étant comptabilisée au bilan de l'investissement que l'étudiant doit faire en vue d'un avenir meilleur. Cependant, il ne faut pas oublier qu'il en résulte souvent un endettement, endettement qui peut avoir des conséquences sur la condition financière au moment de l'insertion professionnelle ou de la formation d'une unité familiale. La dette moyenne des bénéficiaires de l'aide financière aux étudiants était de 5989 \$ en 1991-1992, tous ordres d'enseignement confondus. II va sans dire que les étudiants universitaires de troisième cycle cumulaient la plus forte moyenne, soit 12752 \$ (Direction générale de l'aide financière aux étudiants, 1994 : 4). Mais, en même temps, 
ces derniers constituent le groupe qui a la plus grande probabilité de se trouver un emploi à la fin des études, bien que les études supérieures ne garantissent pas automatiquement une insertion professionnelle rapide et adéquate (Conseil canadien de développement social, 1995).

\section{La pauvreté serait plus grave chez les jeunes, en particulier chez les jeunes qui vivent seuls}

La manière de mesurer la pauvreté qui sous-tend les statistiques montre que les jeunes sont souvent les plus pauvres parmi les pauvres. Dans toutes les catégories de ménage, les jeunes enregistrent un écart plus grand par rapport au seuil que les autres groupes, c'est-à-dire que la proportion de leur revenu par rapport à ce qui est jugé minimal est plus faible que dans les autres groupes d'âge. Ce sont les personnes qui vivent seules et les familles monoparentales qui présentent la situation la plus grave. La proportion de leur revenu se situe à 0,57 et 0,59 respectivement par rapport au seuil fixé pour le groupe auquel ces jeunes se rattachent.

Ces deux groupes attirent particulièrement I'attention. Dans le cas des familles monoparentales la situation ne serait pas irréversible selon les constatations du Conseil national du bien-être social; ainsi, certaines jeunes mères, soit par la mise en couple, soit par le travail lorsque l'enfant ou les enfants leur permettent de s'éloigner du foyer pour occuper un emploi, voient leur situation s'améliorer (Conseil national du bien-être social, 1995 : 35). Force est cependant de constater, comme on le verra plus loin, que ces jeunes familles composent une population nombreuse à l'aide sociale.

Dans le cas des jeunes qui vivent seuls, la situation ne serait pas davantage sans issue, bien que l'ensemble de ces personnes demeurent vulnérables, même aux autres âges de la vie. Les ménages à deux pourvoyeurs connaissent généralement de meilleures conditions que les autres du point de vue de la sécurité financière, même s'ils comptent plus d'un enfant (Langlois, 1994: 19). Le revenu total disponible des personnes seules a connu un écart à la baisse de $10,5 \%$ entre 1978 et 1990 , ce qui en fait le type de ménage qui a connu le changement négatif le plus marqué pendant la période étudiée par l'Enquête sur les dépenses des familles de Statistique Canada (id.).

Si l'on connaît quelque peu le profil des jeunes familles monoparentales (Cournoyer, 1995), celui des jeunes qui vivent seuls est plutôt flou. Chez les moins de 25 ans, les taux sont pratiquement 
aussi élevés chez les jeunes hommes qui vivent seuls que chez les jeunes femmes dans la même condition. Cette situation diffère de celle des autres groupes d'âge où les femmes sont toujours plus nombreuses sous le seuil de faible revenu que les hommes (Conseil national, ibid.: 34). Les femmes de cet âge ont un profil ressemblant sans doute davantage à celui des hommes que dans les autres groupes d'âge où c'est la monoparentalité féminine qui explique la plus grande pauvreté des femmes.

Qui sont les jeunes qui vivent seuls ou en foyer non familial et qui recourent à l'aide sociale? S'agit-il dans ce cas de migrants, les migrations à partir des régions étant particulièrement importantes dans le cas des moins de 30 ans, plus spécialement chez les 20-24 ans (Gauthier et Mercier, 1994: 101) ? Les études du ministère de la Sécurité du revenu sont peu explicites à cet égard, la preuve de la mobilité des jeunes assistés sociaux n'étant pas faite (Lemieux et Lanctôt, 1995: 62). On sait que la proportion des assistés sociaux de 15 à 24 ans vivant avec leurs parents a constamment décru de 1983 à 1993, passant de $68 \%$ à $36 \%$ (Lemieux et Lanctôt, 1995: 63) au moment où l'inverse se produisait dans la population en général depuis le milieu de la décennie de 1980. Les hommes de 21-23 ans, par exemple, étaient $68,8 \%$ à vivre comme enfant dans une famille en 1981 et $71,8 \%$ en 1991 au Canada. Les femmes au même âge étaient $60 \%$ en 1981 et $64,6 \%$ en 1991 (Statistique Canada, 1995: 18) ${ }^{3}$.

Les taux de pauvreté varient aussi selon d'autres caractéristiques: selon la profession du chef de famille, le niveau de scolarité et le sexe. C'est dans le secteur des services que l'on retrouve le plus de travailleurs pauvres et dans celui de la gestion, le moins (Conseil national, 1995: 37). Il y a aussi une proportion plus grande de pauvres chez les personnes peu scolarisées que chez celles qui ont un haut niveau de scolarité. C'est chez les mères seules que l'on retrouve le plus haut niveau de pauvreté, mais aussi le plus bas niveau de scolarité (id.: 40). La maternité en bas âge explique-t-elle le faible niveau de scolarité ? S'agit-il de l'inverse? Selon une étude effectuée à Montréal, la relation entre maternité en bas âge et faible niveau de scolarité est complexe et l'on ne peut déduire aussi rapidement que I'une est la conséquence ou la cause de l'autre (Forget et al., 1992).

Toutefois, les années récentes ont vu s'accroître la proportion de pauvres chez les personnes les plus scolarisées. Le bulletin d'actualité Le Point du Conseil canadien de développement social titre en page couverture: "Instruits mais pauvres» (1995: 1). Bien que ces nouveaux pauvres soient proportionnellement plus nombreux chez les moins de 25 ans (16\% par rapport à $10 \%$ dans l'ensemble de la 
population) (Conseil canadien de développement social: 2), ils ne se recrutent pas uniquement chez les jeunes, mais se trouveraient à tous les âges et auraient les caractéristiques les plus variées. L'auteur de l'étude souligne que "le diplôme postsecondaire ne peut garantir un emploi, encore moins un emploi avec un bon salaire" (ibid.). Il s'agirait moins de la qualité de la formation qui serait en cause que de l'absence de bons emplois disponibles.

Une nouvelle catégorie de pauvres a fait son apparition au cours des dernières décennies, non pas nouvelle dans sa forme, mais parce qu'elle atteint des personnes de plus en plus jeunes un peu partout dans les pays industrialisés. Cette catégorie, l'itinérance, est à l'origine de plusieurs manières d'analyser le phénomène de la pauvreté. Plusieurs, empruntant en cela le terme à la sociologie française, ont parlé d'exclusion sociale. Shirley Roy préfère, pour sa part, qualifier ce phénomène de "désinsertion sociale " et conserver I'appellation $d^{\prime}$ exclusion pour marquer "la limite ultime d'un long processus" (1995: 74). On observe une certaine unanimité quant à l'augmentation et à la plus grande visibilité du phénomène, difficile à quantifier. Un relevé effectué entre le $1^{\text {er }}$ mars 1988 et le 22 février 1989 indique qu'il y aurait eu 8756 personnes hébergées dans les refuges de la ville de Montréal, dont $40 \%$ environ auraient eu moins de 30 ans (Chalom et Kousik, 1992: 56). À Québec, il y aurait une centaine (davantage l'été) de jeunes de 12 à 20 ans qui vivent dans la rue le jour, mais entrent chez eux le soir pour dormir et la clientèle des Soupes populaires rajeunirait (Le Soleil, 4 avril 1993: B-1 et $1^{\text {er }}$ octobre 1995 : B-3). Faut-il attribuer ce fait aux difficultés d'insertion professionnelle, au malaise ressenti par certains jeunes à l'égard de leur famille, à une manière nouvelle d'affirmer son identité? S'agitil d'un phénomène de groupe, de classe, qui pourrait s'apparenter à celui des "vestes de cuir " à la fin des années 60 qui exprimaient, à leur façon, leur distance par rapport aux valeurs de la société? L'itinérance, par sa visibilité, mais en même temps par l'ambiguïté de sa signification, remet en question les différentes définitions de la pauvreté et, surtout, les explications qui en sont données.

\section{Parce qu'ils sont plus pauvres, les jeunes sont-ils davantage "dépendants " de l'aide sociale?}

Une confusion règne quant à l'utilisation du mot "dépendance " en ce qui concerne l'aide sociale. Le taux de bénéficiaires de I'aide sociale se définit comme un "taux de dépendance». Celui-ci s'obtient «en divisant le nombre d'adultes de ce groupe bénéficiant 
de l'aide de dernier recours par le nombre total de personnes de ce groupe dans la population " (Lemieux et Lanctôt, 1995: 33). Ce taux qui n'indique qu'une proportion laisse pourtant planer, à cause de sa connotation négative, l'idée de "durée" de présence à l'aide sociale. Entendant le mot "dépendant", le glissement est facile à faire: de la notion d'inscription - qui peut n'être que temporaire ou passagère - à celle de "carrière " que la dernière étude publiée par le ministère de la Sécurité du revenu suggère par son titre, Commencer sa vie adulte à l'aide sociale, le pas est vite franchi.

Contrairement à une opinion répandue, tous les jeunes pauvres ne $s^{\prime}$ inscrivent pas à l'aide sociale, puisque $17 \%$ des moins de trente ans sont soit bénéficiaires de l'assurance-chômage, soit bénéficiaires de I'aide sociale (Lemieux et Lanctôt, 1995: 30) qualifiée d'aide de dernier recours depuis la réforme de 1988. De ce nombre, $43 \%$ reçoivent de l'assurance-chômage et $57 \%$ de l'aide sociale. Contrairement à une autre opinion répandue, de 1975 à 1994, soit sur une période de vingt ans, pendant sept ans seulement les jeunes ont été proportionnellement plus nombreux que les 30-64 ans à s'inscrire à l'aide sociale. Or, il se trouve que ces années se répartissent sur deux périodes, soit de 1983 à 1986 et de 1992 à 1994, là où les taux de chômage ont été les plus élevés depuis la grande crise (MSSS, 1995 : 82).

II existe une relation entre les taux de chômage et les taux de pauvreté, les taux de chômage et le recours à l'aide sociale (Lemieux et Lanctôt, 1995: 33). Les jeunes sont particulièrement à risque de chômage et ils forment la catégorie la plus à risque d'intermittence en emploi. Des analystes n'hésitent pas à qualifier le marché du travail de marché "en transition» (Conseil national du bien-être social, 1993: 5), d'autres, de marché fluctuant et particulièrement à risque pour les jeunes (MSSS, 1995: 285). En dépit de ces difficultés particulières, le taux d'inscrits à l'aide de dernier recours n'est guère plus élevé chez les moins de 30 ans que chez les 30-64 ans : 12,4\% pour les premiers et $11,1 \%$ pour les autres en 1994 . Plus que cela, ce que les analystes nomment "le taux de dépendance globale», qui s'obtient en additionnant le taux de dépendance à l'aide sociale et le taux de dépendance à l'assurance-chômage (Lemieux et Lanctôt, 1995: 37), montre un transfert de l'assurance-chômage à l'aide sociale. Ce n'est donc pas "le refus du travail » (Lemieux et Lanctôt, 1995 : 37) qui explique l'augmentation du taux de bénéficiaires de I'aide sociale à certaines périodes au cours des dernières décennies, mais un resserrement des règles d'attribution de l'assurance-chômage dans un contexte pourtant particulièrement difficile. 
Le taux de présence à l'aide sociale des jeunes femmes est plus stable que celui des jeunes hommes. Les fluctuations laissent deviner la présence de facteurs explicatifs différents selon le sexe. Ainsi, en période de reprise économique, le fossé se creuse, les jeunes hommes étant davantage atteints par des facteurs conjoncturels (les récessions). Les jeunes femmes recourent à l'aide sociale le plus souvent pour des raisons structurelles (la monoparentalité) (Lemieux et Lanctôt, 1995: 35). Cette raison explique qu'elles soient les bénéficiaires les plus stables et pour des durées plus longues. Une comparaison de trois cohortes à des moments différents, 1983, 1989 et 1993, indique une augmentation des jeunes parents : « $88 \%$ des jeunes assistés sociaux étaient sans enfants à charge en 1983 contre $77 \%$ en 1993 » (Lemieux et Lanctôt, 1995: 63).

Ce qui étonne le plus en parcourant les données publiées sous l'égide du ministère de la Sécurité du revenu, c'est l'absence quasi totale d'information concernant les raisons d'entrée à l'aide sociale. Le cafouillis dans la manière de recueillir l'information s'amplifie avec le temps. Ainsi, en 1983, la catégorie "autres» constituait $45,7 \%$ de l'ensemble des raisons données par les jeunes de moins de 21 ans pour expliquer leur présence à l'aide sociale, alors qu'en 1989 elle était de 55,0\% et en 1993, de 57,9\% (Lemieux et Lanctôt, $1995: 61$ ).

Les données statistiques mettent ainsi sur des pistes, permettent d'éliminer au départ des hypothèses fausses ou des représentations inadéquates, mais elles laissent aussi sur des interrogations. La manière de recueillir les données constitue elle-même un reflet sélectif qu'il faut constamment remettre en question et qui ne doit pas laisser dans l'ombre le point de vue des acteurs eux-mêmes.

\section{DES POINTS D'OMBRE ET DES QUESTIONS}

La pratique traditionnelle de la sociologie repose sur l'utilisation - et la production - de ces données brutes, de ces mesures dites conformes de la réalité sociale pour une période délimitée. Or, après d'autres, mais avec plus d'autorité, Anthony Giddens renouvelle un avertissement servi naguère aux positivistes: les données statistiques constituent elles-mêmes un produit social (1990: 42). Ces informations chiffrées sur les caractéristiques d'une population, de ménages ou de particuliers sont en partie tributaires du découpage catégoriel retenu et de la réaction plus ou moins consciente des sujets répertoriés à ces mêmes informations régulièrement communiquées. Ainsi la sélection et la définition des caractéristiques d'une population répondent d'abord à des besoins administratifs. De plus, les segments de 
population circonscrits de la sorte sont, en retour, sensibles aux données qui les concernent et qui font les manchettes des journaux et l'objet de débats télévisés. Les jeunes voient étalées leur quote-part de chômage, de pauvreté, de célibat, d'isolement résidentiel, etc., et leur probabilité d'y aboutir ou de s'y enliser.

Les observations quotidiennes montrent alors les limites du tableau brossé de la vie des jeunes vivant sous le seuil de faible revenu. Les statistiques, même confrontées les unes aux autres dans un effort de les faire discourir selon les derniers raffinements de I'analyse quantitative, demeurent muettes sur des variantes autrement appréhendées de l'existence des jeunes. De quels cheminements, stratégies et conditions sont tissées ces amorces de vie autonome? Vivre avec peu de ressources entre 15 et 24 ans, voilà une donnée préliminaire qui s'éclate en une diversité de situations. Du montant minimal alloué par la Sécurité du revenu à ce groupe d'âge au seuil de faible revenu, divers degrés d'accommodement ou d'indigence peuvent être entrevus. Et entre résider seul et vivre isolé ${ }^{4}$ la nuance est significative et les implications économiques et psychosociales se révèlent considérables.

\section{Comment repérer la diversité des situations ou la gamme des possibles?}

Il y a d'abord une faiblesse inhérente aux statistiques sur les revenus : toutes les sources ne sont pas connues et certaines peuvent difficilement être comptabilisées, en particulier dans le cas des jeunes où les liens avec la famille d'origine peuvent être maintenus pendant un certain temps en dépit d'une séparation apparente. De même, un pourcentage incertain de jeunes - à l'instar des aînés - pourraient "ajuster" leurs réponses aux formulaires des recenseurs. On tairait les revenus du travail au noir que ceux-ci ajoutent à des émoluments modestes d'emploi ou aux maigres prestations de la Sécurité du revenu. Ainsi, à l'étalement des revenus déclarés, et à la variété et fiabilité de leur provenance (emploi, assurance-chômage, prestations sociales), s'adjoignent des compléments pécuniaires ou tangibles qui augmentent d'autant la diversité des situations concrètes d'existence. Or, les apports matériels (nourriture, vêtements, meubles) d'organismes communautaires, religieux ou caritatifs (banques alimentaires, soupes populaires, coupons d'alimentation, procures et vestiaires paroissiaux, etc.) ou obtenus gracieusement de parents ou d'amis ne figurent pas, non plus, dans les déclarations de revenu. Ils permettent parfois de faire la différence entre vivre dans l'indigence et vivre dans une situation qui $n^{\prime}$ entache pas la dignités. 
De même et de surcroît, le statut résidentiel déclaré ne correspondrait pas toujours au mode d'habitation réel. L'option de la colocation semble retenue par certains jeunes comme une formule d'appoint efficace. L'incertitude de ce statut découle de la mobilité caractéristique des jeunes. L'ancrage et la stabilité résidentielle requièrent un minimum de garanties professionnelles et $d^{\prime}$ 'engagement relationnel. Dans le laps d'une année, au gré des aléas de l'emploi, de la rupture des liens amoureux ou amicaux, ou de la surveillance des agences sociales, les ententes d'hébergement ou de partage résidentiel se font, se défont et se succèdent. Mais au-delà d'une possible épargne grâce au partage du coût du loyer et des frais afférents, c'est la disponibilité d'un réseau social additionnel qui intéresse: celui d'un colocataire. Car l'interrogation sous-jacente ici porte sur l'effet éventuellement structurant de l'effritement des liens sociaux sur le processus de l'appauvrissement. Une double instabilité (et partant précarité) règne dans l'univers des jeunes. Celle de l'insertion et du maintien en emploi qui gouvernent l'autonomie financière et celle des rapports sociaux noués dans les contextes de la famille, de l'école, du travail et du loisir, et par la voie desquels transite parfois l'accès à l'emploi. Ainsi apparaît-il opportun de se pencher sur l'action de ces réseaux. Quelle peut être leur efficacité, compte tenu de leur diversité et de leur endurance variables sur la quête d'emploi ou la bonification des conditions matérielles d'existence? On pourrait aussi s'interroger sur le rôle des organismes communautaires dans l'intégration sociale, un espace de solidarité où les jeunes peuvent partager la même expérience de pauvreté et $d^{\prime}$ isolement. Ce peut être aussi un espace de résistance où ils donnent un sens à leur vie et élaborent des pratiques autres (White, 1994).

Que les jeunes habitent seuls à longueur d'année ou non, qu'ils bénéficient de revenus en deçà, au-delà ou à la marge de la suffisance avec l'ajout ou non d'expédients ou de l'entraide assortie aux réseaux de sociabilité, il demeure que cette diversité de conditions pécuniaires et matérielles ne peut seule servir à délimiter des catégories de jeunes pauvres. Pour construire des «types" de jeunes en situation dite de pauvreté, il faut intégrer à cette construction les cheminements qui y ont mené et les stratégies arrêtées de débrouille immédiates ou projetées dans l'avenir.

\section{Les chemins mal connus de l'autonomie}

Le discours sociopolitique le plus courant loge le malaise économique des jeunes à la croisée des avatars de la restructuration du marché du travail, de l'école et de la famille. Sans succomber aux récriminations 
idéologiques, il s'impose d'examiner le parcours effectué par les jeunes dans les milieux qui leur sont plus particulièrement réservés. De quels actifs acquis au long de ce parcours disposent les jeunes au moment où ils amorcent une existence autonome? Sont-ils toujours effectivement autonomes lorsqu'ils choisissent d'exercer globalement leur indépendance (ou sont forcés de le faire) ? Les acquis présumés des jeunes relèvent du potentiel du milieu d'origine et de leur rapport aux effectifs du milieu de socialisation. La famille, pour sa part, sert de point $d^{\prime}$ ancrage polyvalent. Elle ouvre sur des réseaux de parenté, de voisinage et d'amitié, elle dispose à divers degrés de ressources financières ou matérielles et propose, par ses pratiques ou ses attentes, des projets de vie professionnelle et sociale. De la richesse et de la pertinence conjoncturelle de ces apports et de l'aptitude de la famille à les transmettre dépend la préparation de la jeune génération à s'insérer dans la vie active.

Or, si la qualité de la relation familiale se reflète dans la valeur du patrimoine reçu en partage par le jeune qui quitte le foyer parental, les effets de la relation débordent largement ce départ. Les bons ou mauvais termes dans lesquels se sont entretenus jeunes et parents sont susceptibles de colorer les rapports ultérieurs. Les raisons qui ont suscité le départ (parfois hâtif) des jeunes laissent-elles préjuger de la volonté des parents à contribuer, besoin étant, à la subsistance de leur jeune adulte? La mésentente, la violence familiale, les difficultés financières des parents, l'incitation des pairs, la dégradation du marché local du travail, etc., affectent-elles de même manière les prédispositions parentales? Le soutien de type pécuniaire, lorsqu'il est consenti et accepté, s'accompagne-t-il d'aide matérielle ou de soutien affectif sans égard aux conditions de départ?

Pour mieux saisir le regard porté par les 16-24 ans sur leur propre condition, il importe aussi de répertorier les stratégies plus ou moins articulées et réfléchies qu'échafaudent les jeunes pour assurer leur survie et leur bien-être matériel. La conception de stratégies opérantes, à court ou moyen terme, repose sur la connaissance des multiples voies d'accès, directes ou indirectes, aux biens et services essentiels. L'utilisation judicieuse des ressources d'un quartier, l'acceptation du recours aux programmes sociaux et des contraintes associées ne représentent que deux des constituantes possibles d'un plan concerté. Le retour aux études allié au travail à temps partiel ou intercalé entre des épisodes d'activité rémunérée constitue une autre variante de la débrouille. Le maniement adroit du potentiel des réseaux sociaux débouche aussi sur des informations privilégiées, sur des offres d'hébergement et de sustentation alimentaire et sur l'obtention de petits boulots plus ou moins parallèles ou avouables. 
Ainsi, I'habileté à sélectionner et à combiner des stratégies prometteuses devrait permettre de mieux cerner les perspectives d'avenir de ce groupe d'âge. Pour cela, il faut pouvoir départager, dans les projets formulés, les discours qui reflètent surtout le désir de ceux qui prennent appui sur des démarches déjà amorcées.

Par le moyen d'une analyse discriminante, il serait envisageable de circonscrire les pratiques socio-économiques et les représentations propres à cette génération. L'examen des bribes de vie livrées et des représentations de la situation actuelle et de celles de l'avenir devrait permettre de déceler chez les jeunes la persistance ou le renversement des modalités d'intégration professionnelles et sociales pratiquées par la génération antérieure. Cette analyse permettrait aussi d'éviter de concevoir une situation temporaire difficile comme une situation marginale ou conduisant à la marginalité. Dans un article récent, Olivier rappelle fort à propos: "On est en effet parfois trop rapidement tenté $\mathrm{d}^{\prime}$ assimiler l'ensemble des situations de transition à des situations d'exclusion» (1996: 191).

\section{Les théories sociologiques actuelles permettent-elles de penser différemment la pauvreté chez les jeunes?}

Le phénomène de la pauvreté a été analysé selon différentes perspectives reflétant les préoccupations théoriques et sociales de l'heure. À partir des théories sociologiques plus couramment utilisées pour aborder la pauvreté et ses représentations, quels sont les éléments qui peuvent être sélectionnés et articulés pour penser différemment la pauvreté des jeunes dans ses manifestations? En d'autres mots, quels éléments permettraient de donner la parole aux jeunes et, ainsi, de faire ressortir la complexité du phénomène? Cet arrêt sur les acquis statistiques et les acquis théoriques a pour objectif d'opérer un premier filtrage parmi les théories pertinentes des éléments les plus prometteurs pour une compréhension renouvelée du phénomène actuel de la pauvreté chez les jeunes.

Dans l'après-guerre, en dépit de l'essor économique, des poches de pauvreté persistante ont continué à faire problème ou à déranger. L'approche fonctionnaliste et, dans sa foulée, l'approche épidémiologique ont été privilégiées comme mode d'explication et de résolution de ce qui était considéré comme anomalie. En somme, la perspective fonctionnaliste envisage la pauvreté comme un problème social qui découlerait de conduites déviantes, non conformes aux normes sociales établies. L'acteur ne fait pas siennes les caractéristiques du modèle intégrateur. Dans cette même perspective, 
I' "approche à risque», issue de l'épidémiologie et largement répandue dans le réseau de la santé et des services sociaux, conçoit la pauvreté comme une pathologie. Elle vise essentiellement à identifier "les conditions d'apparition de comportements non désirés et à agir sur les déterminants environnementaux» (Parazelli, 1995: 25) susceptibles d'influencer l'augmentation du taux de pauvreté chez certains individus ou groupes. Ainsi, on tend à désigner chez les jeunes ceux qui correspondent au profil de déviance: les fugueurs, les décrocheurs, les jeunes de la rue, les itinérants, etc. La composante retenue de la pauvreté des jeunes serait leur conduite marginale.

Un second mouvement théorique, celui des théoriciens du conflit, s'insurge contre cette vision qui attribue la pauvreté au pauvre lui-même, qui rend responsables les victimes de la distribution inégale des ressources et du pouvoir. La pauvreté du jeune, présentée comme un échec personnel par les fonctionnalistes, est attribuée ici exclusivement à des déterminants sociaux qui appellent à une macroanalyse des "formations sociales et économiques». Dans ce cadre, est évacuée la contribution dynamique de l'acteur à la construction de «son monde».

Quant à l'interactionnisme symbolique, il repose plutôt sur I'idée que l'ordre social est construit à travers l'évolution de la signification. En effet, il postule que la vie en société implique un processus d'interaction, d'où la possibilité d'entretenir des visions explicatives divergentes de la pauvreté. Et cela, à partir du pôle où I'on se trouve dans I'interaction. Dans cette optique, le pauvre peut s'opposer ou adhérer aux diverses définitions qui circulent dans la société à son sujet: paresse, absence d'initiative, indifférence ou hostilité à l'éthique du travail, tout autant que "victime» de la conjoncture ou de l'égoïsme générationnel, etc. Cette interprétation peut être intériorisée au point que le pauvre s'attribue l'identité qui lui est conférée.

Dans un effort de comprendre la tension dialectique, Dubet, dans ce qu'il nomme "sociologie de l'expérience», propose "une combinaison de logiques d'action, logiques qui lient l'acteur à chacune des dimensions d'un système. L'acteur est tenu d'articuler trois logiques $d^{\prime}$ action et $c^{\prime}$ est la dynamique engendrée par cette activité qui constitue la subjectivité de l'acteur et sa réflexivité " (Dubet, 1994: 105). La première logique, celle de l'intégration sociale, concerne la façon dont les jeunes pauvres, dans le cas qui nous intéresse, se définissent par rapport à leurs appartenances. La société est alors perçue comme un système d'intégration. Dans la logique stratégique, le jeune cherche ses intérêts, c'est-à-dire les moyens (tactiques de débrouillardise) ou les ressources de son 
environnement qui lui permettront d'assurer son existence ou même, dans certains cas, sa survie. Dans la troisième logique, "l'acteur se représente comme un sujet critique confronté à une société définie comme un système de production et de domination " (Dubet, $1994: 111)$.

\section{CONCLUSION}

Les projets à venir pourraient prendre en considération cette dimension du réel que veulent réfléchir les mesures objectives de la pauvreté. Toutefois, comme ces mesures n'épuisent pas la réalité du phénomène, l'étape suivante consiste à observer l'articulation ou la combinaison des stratégies adoptées par divers types de jeunes. Le choix des stratégies par ce groupe d'âge demeure tributaire des ressources connues et disponibles ainsi que fonction des contraintes dans lesquelles les jeunes aujourd'hui se débattent. En somme, les bouleversements du contexte économique et social obligeraient les jeunes à de nouvelles façons de percevoir leur situation et d'agir en conséquence, transformant ici ce qui, jusqu'à maintenant, pouvait être considéré comme "la normalité » dans le monde du travail et la vie relationnelle. Le rôle du chercheur n'est-il pas de laisser l'acteur se décrire dans son cheminement tout en le resituant dans son univers sociétal?

\section{Notes}

1. Nous avons nous-mêmes publié un bilan sur cette question, qui montre un certain nombre de limites dans les connaissances actuelles en ce qui touche la pauvreté des jeunes (Gauthier et Mercier, 1994). Un projet en cours soulève des interrogations sur la place et l'importance du lien social dans un contexte de précarité chez les jeunes. Un certain nombre de questions posées ici nourrissent la problématique à l'origine de cette recherche financée par le Conseil québécois de la recherche sociale.

2. Nous suggérons la lecture d'un article de Jean-François René sur " la place des ressources intermédiaires" dans un contexte de vie précaire (1994 : 151-161).

3. Des chercheurs français vont jusqu'à s'interroger sur une possible solidarité de ceux qui quittent la famille lorsque celle-ci connaît des difficultés financières, évitant ainsi d'être à sa charge (Blöss et al., 1990 : 556).

4. Louise Saint-Laurent confronte ces deux réalités dans une étude du phénomène social de la solitude à travers l'expérience des néo-célibataires (1993).

5. Andrée Roberge a déjà montré comment l'échange informel peut faire toute la différence entre vivre dans la pauvreté ou pas (1987). 


\section{Références bibliographiques}

BLÖSS, T., A. FRICKEY et F. GODARD (1990). "Cohabiter, décohabiter, recohabiter» Revue française de sociologie, vol. XXXI : 553-572.

CHALOM, M. et J. KOUSIK (1993). Violence et déviance à Montréal. Montréal : Liber.

COnSEIl CANadien de développement social (1995). Le Point, $\mathrm{n}^{\circ} 2$, juin.

CONSEIL NATIONAL DU BIEN-ÊTRE SOCIAL (1993). Choisir de travailler: incitatifs et désincitatifs : rapport. Ottawa.

CONSEIL NATIONAL DU BIEN-ÊTRE SOCIAL (1995). Profil de la pauvreté, 1993 : rapport. Ottawa.

CONSEIL PERMANENT DE LA JEUNESSE (1993). "Dites à tout le monde qu'on existe...", Avis sur la pauvreté des jeunes. Québec: Gouvernement du Québec.

COURNOYER, M. (1995). Grossesse, maternité et paternité à l'adolescence: recueil de recension des écrits. Québec: IQRC.

DIRECTION DE L'AIDE FINANCIÈRE AUX ÉTUDIANTS (1994). Étude sur la dette accumulée par les bénéficiaires de l'aide financière aux étudiants. Québec: Ministère de l'Éducation.

Dubet, F. (1994). Sociologie de l'expérience. Paris: Seuil.

FORGET, G., A. BILODEAU et J. TÉTREAULt (1992). "Facteurs reliés à la sexualité et à la contraception chez les jeunes et décrochage scolaire: un lien insolite mais réel ", Apprentissage et socialisation, vol. 15, n 1 : 29-38.

Galland, O. (1996). "Les jeunes et I'exclusion», dans Serge Paugam (dir.), L'exclusion: l'état des savoirs. Paris: La Découverte, p. 183-192.

GAUTHIER, M. (1994). Une société sans les jeunes? Québec: IQRC.

GAUTHIER, M. et L. MERCIER (1994). La pauvreté chez les jeunes: précarité économique et fragilité sociale: un bilan. Québec: IQRC.

GIDDENS, A. (1990). The Consequences of Modernity. Standford: Standford University Press.

LANGLOIS, S. (1994). "Le niveau de vie des familles: déclassement et effet de génération», dans G. Pronovost (dir.), Comprendre la famille. Sainte-Foy: Presses de I'Université du Québec, p. 17-30.

LEMIEUX, N. et P. LANCTÔT (1995). Commencer sa vie adulte à l'aide sociale. Québec: Ministère de la Sécurité du revenu.

MINISTÈRE DE LA SANTÉ ET DES SERVICES SOCIAUX (1995). Le Québec comparé: indicateurs sanitaires, démographiques et socio-économiques. Québec.

PARAZELLI, M. (1995). "De la pauvreté traitée comme une maladie", Le Monde diplomatique, décembre. Paris.

RAVANERA, Z.R. (1995). "Portrait de la vie familiale des jeunes adultes", dans R. Beaujot et al., La famille au long de la vie. Ottawa : Statistique Canada: 9-39 (cat. 91-543-S, hors-série).

RENÉ, J.-F. (1994). "Jeunes adultes et vie précaire : la place grandissante des ressources intermédiaires", Lien social et Politiques - RIAC, $\mathrm{n}^{\circ} 32$ : $151-161$. 
ROBERGE, A. (1987). "L'échange informel en milieu semi-rural pour faire face à la pauvreté ", dans Madeleine Gauthier (dir.), Les nouveaux visages de la pauvreté. Québec: IQRC, p.173-196 (Questions de culture, $\left.n^{\circ} 12\right)$.

RoY, S. (1995). "L'itinérance: forme exemplaire d'exclusion sociale? », Lien social et Politiques - RIAC, $\mathrm{n}^{\circ} 34$ : 73-80.

SAINT-LAURENT, L. (1993). "La dynamique de la solitude des néo-célibataires: vers la constitution de nouveaux réseaux de solidarité ", Lien social et Politiques - RIAC, $\mathrm{n}^{\circ} 29: 151-161$.

WHITE, D. (1994). "La gestion communautaire de l'exclusion », Lien social et Politiques - RIAC, $\mathrm{n}^{\circ} 32: 37-51$. 